\title{
Current National and International Guidelines for the Management of Male Hypogonadism: Helping Clinicians to Navigate Variation in Diagnostic Criteria and Treatment Recommendations
}

\author{
Ahmed Al-Sharefi ${ }^{1}$, Richard Quinton ${ }^{1,2}$ \\ ${ }^{1}$ Department of Endocrinology, Newcastle Upon Tyne Hospitals NHS Foundation Trust; ${ }^{2}$ Translational \& Clinical Research \\ Institute, Newcastle University, Newcastle-upon-Tyne, UK
}

Male hypogonadism — rebadged by some as testosterone deficiency syndrome — is a clinical and biochemical diagnosis of increasing worldwide interest. Organic male hypogonadism — usually permanent - is well-established, but aging men may also exhibit lower serum testosterone levels; principally due to burden of extra-gonadal comorbidities such as obesity, diabetes and metabolic syndrome, but with an underlying intact hypothalamo-pituitary-testicular (HPT) axis capable of springing back into operation once comorbidities are addressed. Despite encouraging observational data and plausible theoretical underpinning, evidence for efficacy and safety of testosterone in this "aging" group of men is lacking; addressing comorbid illnesses remains the key priority instead. Nevertheless, in recent years, accumulation of misleading information online has triggered a global tsunami of testosterone prescriptions. Despite this, many men with organic hypogonadism remain undiagnosed or untreated; many more face a diagnostic odyssey before achieving care by the appropriate specialist. As testosterone therapy is not without risk several clinical practice guidelines have been published specialist societies to guide physicians on best practice. However, these are heterogeneous in key areas, reflecting divergent approaches to the same evidence basis. Herein, we navigate the major clinical practice guidelines on male hypogonadism and test their respective recommendations against current best evidence.

Keywords: Hypogonadism; Testosterone; Sexual dysfunction; Practice guideline; Male aging

\section{INTRODUCTION}

Male hypogonadism ( $\mathrm{MH}$ ) - termed testosterone ( $\mathrm{T}$ ) deficiency syndrome by some investigators - is a clinical syndrome characterized by impaired testicular function, with reduced or absent spermatogenesis and $\mathrm{T}$ secretion. It is caused either by disease

Received: 7 July 2020, Revised: 23 July 2020, Accepted: 27 July 2020

Corresponding author: Richard Quinton

Department of Endocrinology \& Diabetes, The Royal Victoria Infirmary,

Newcastle Upon Tyne Hospitals NHS Foundation Trust, Queen Victoria Rd,

Newcastle upon Tyne NE1 4LP, UK

Tel: +44-191-282-4635, Fax: +44-191-233-6161

E-mail: Richard.Quinton@ncl.ac.uk at the level of the hypothalamus or pituitary gland (central, secondary, or hypogonadotropic hypogonadism $[\mathrm{HH}]$ ), wherein the testes lack gonadotropic stimulation by luteinizing hormone (LH) and follicule stimulating hormone, or an intrinsic defect of the testes themselves (primary hypogonadism $[\mathrm{PH}]$ ), wherein the function Leydig and Sertoli and germ cells is intrinsically

\section{Copyright $(92020$ Korean Endocrine Society}

This is an Open Access article distributed under the terms of the Creative Commons Attribution Non-Commercial License (https://creativecommons.org/ licenses/by-nc/4.0/) which permits unrestricted non-commercial use, distribution, and reproduction in any medium, provided the original work is properly cited. 
impaired (Fig. 1) [1].

The prevalence of MH has been estimated at $6 \%$ to $12 \%$, depending on the study population and the degree of diagnostic rigor $[2,3]$. If untreated, $\mathrm{MH}$ can lead to sexual dysfunction, anemia, osteoporosis and fracture, myopathy and frailty, tender gynecomastia, psychosocial impairments and reduced quality of life. There is also a strong association of MH with obesity, metabolic syndrome and type 2 diabetes mellitus (T2DM) [4], although the direction of causation is unclear [5].

Cross-sectional and longitudinal studies have generally shown a decline in serum $\mathrm{T}$ levels with advancing age, in parallel to increasing mortality [6,7], leading some investigators to coin the term "late-onset hypogonadism" (LOH). The European Male Ageing Study (EMAS) observed serum $\mathrm{T}<8 \mathrm{nmol} / \mathrm{L}$ $(<230.5 \mathrm{ng} / \mathrm{dL})$ to be associated with increased all-cause and cardiovascular (CV) mortality [6]. However, EMAS also found the overwhelming contribution to the apparent age-related decline in $\mathrm{T}$ levels to reside not in chronological aging per se, but rather in the accumulation of age-associated co-morbidities, such as obesity, resulting in suppression of LH secretion [8]. This being a reversible physiological phenomenon, it is perhaps more accurately described as "non-gonadal illness" (NGI) and is likely distinct from organic $\mathrm{HH}[9,10]$. Hence, questions of diagnostic specificity apply to EMAS and many other studies that describe populations of older, obese men with slightly low $\mathrm{T}$ levels as having adult-onset HH. Indeed, although the Survey on Prevalence in East China for Metabolic Diseases and Risk Factors (SPECT)-China study found a similar fall in T levels to EMAS up to and including middle age, older men who had maintained traditional non-Western diet and largely avoided weight-gain did not exhibit lower $\mathrm{T}$ levels than younger Chinese men [11]. An Australian study of fit, healthy community-dwelling older men likewise found similar $\mathrm{T}$ levels to younger men [12]. Nevertheless, both EMAS and SPECT-China reported organic $\mathrm{PH}$ in $1 \%$ to $2 \%$ of older men (although EMAS curiously devised an entirely new term "high LH-hypogonadism" to describe $\mathrm{PH}$ ), with another $10 \%$ of men having "compensated" $\mathrm{PH}$. Compensated PH is a purely biochemical descriptor, unrelated to clinical phenotype, wherein LH is elevated yet T remains within reference limits. Nevertheless, for any man having "compensated $\mathrm{PH}$ " and clinically-ascertained anemia, osteoporosis, or refractory sexual dysfunction, the question necessarily

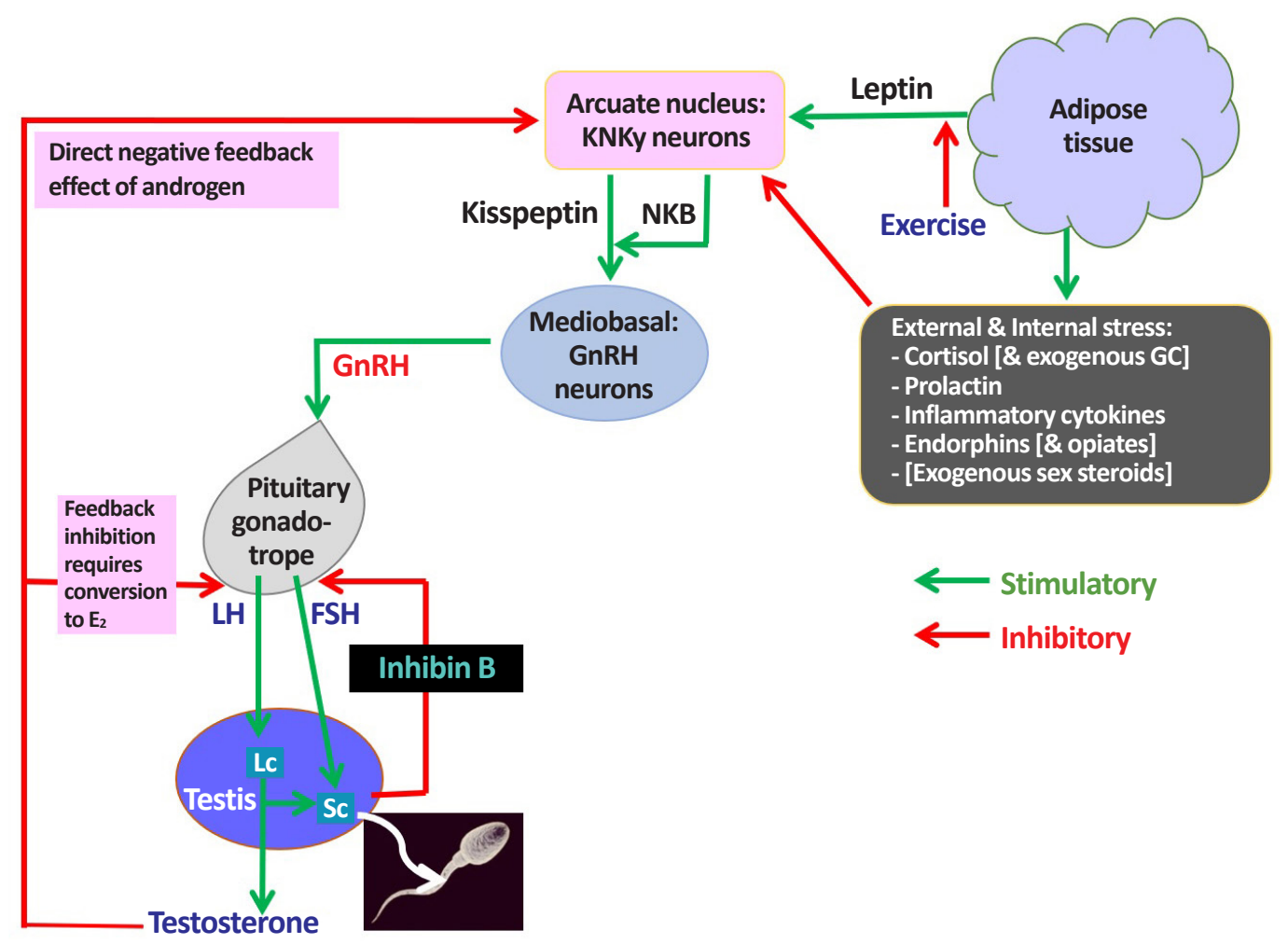

Fig. 1. Male hypothalamic-pituitary-gonadal (HPG) axis: homeostatic \& environmental inputs: endocrine \& paracrine actions of testosterone. KNDy, kisspeptin, neurokinin B, and dynorphin; NKB, neurokinin B; GC, glucocorticosteroid; GnRH, gonadotropin-releasing hormone; E2, oestradiol; FSH, follicle-stimulating hormone; LH, luteinizing hormone; Lc, Leydig cell; Sc, Sertoli cell. 
arises, "just how compensated is that from a clinical perspective?" It is thus particularly for these men, among whom PH was associated in equal measure with chronological age and burden of co-morbidities, that the term LOH most appropriately applies [13]. Intriguingly, men with PH have a 3 to 4-fold relative risk of having T2DM, whether related to ageing [11], Klinefelter syndrome [14], or myotonic dystrophy [15].

Over the past 20 years, there has been a worldwide surge of $\mathrm{T}$ prescribing, mainly for age- or obesity-related decline in serum $\mathrm{T}$ and/or men reporting erectile dysfunction. In the United States, testosterone prescribing has tripled from 2001 to 2011. Furthermore, many have been prescribed testosterone without any prior testing of serum $\mathrm{T}$ level, or even more worryingly with normal initial T level [16,17]. In the United Kingdom, there was a $90 \%$ increase in testosterone prescribing from 2001 to 2010 that could not be satisfactorily explained [18]. Similar unjustified trends in testosterone prescribing were observed in Australia over a 20-year period [19]. Whilst it is conceivable that genuine need is now being addressed that was previously unmet, these observations more plausibly reflect the impact of promotional and educational activity to portray testosterone as an "elixir" of sexual and cardio-metabolic youth. This is additionally overlaid by increasing observations of "testosterone seeking behavior' (TSB) by vulnerable men whose views have been influenced by unregulated "men's health" websites and online chatrooms [20].

Although the benefits and safety profile of using testosterone to treat well-found $\mathrm{MH}$ are well-established, its use in men without a verified diagnosis of hypogonadism carries significantly less reassurance [21]. In fact, older or obese men receiving testosterone therapy outside the umbrella of organic hypogonadism may be at risk of developing secondary polycythemia, CV disease and detection of subclinical prostate cancer $[22,23]$, not to mention androgen-induced hypogonadotropic infertility.

Sexual medicine has an extraordinarily low profile in medical school curricula worldwide, with the relevant Multidisciplinary Joint Committee of European Union of Medical Specialists (UEMS) having written to the European Commission in 2019 asking for "at least 1 hour of training" to be mandated across European medical schools. Even among doctors undergoing postgraduate training in endocrinology, urology, or family/primary care medicine, exposure to dedicated training in sexual medicine is of variable granularity.

To sign-post clinical practitioners to men most likely benefit from testosterone treatment, several national and international societies have produced clinical practice guidelines for the diagnosis and management of MH. However, recommendations necessarily reflect the experience, interests and standard operating procedures of the specialty concerned and, hence these guidelines are not always consistent with each other in key areas. As a result, mainstream physicians lacking experience in managing men presenting with symptoms potentially related to $\mathrm{MH}$ may be left confused, or uncertain of what to do for the best.

In this review, we compare and contrast all the national and international guidelines for the diagnosis and management of $\mathrm{MH}$, namely: 2015 The International Society for Sexual Medicine (ISSM) [24], 2017 The British Society for Sexual Medicine (BSSM) [25], 2015 The Canadian Medical Association Journal (CMAJ) [26], 2018 The American Urological Association (AUA) [27], 2018 European Association of Urology (EAU) [28], 2018 Endocrine Society (ES) [1] and, in 2016, The Endocrine Society of Australia position statement on MH (AUS) [29,30].

In exploring the major differences and similarities in their respective recommendations, we highlight how these variations might differentially impact clinical practice and discuss the therapeutic and pharmaco-economic implications thereof. We also outline how, in reviewing these different guidelines, we were motivated to achieve a synthesis of international best practice relating to $\mathrm{MH}$ that was both simple and rigorous enough to guide clinical practice for primary care physicians working within the United Kingdom's National Health Service (NHS) (Fig. 2).

\section{DEFINING MH CLINICALLY AND BIOCHEMICALLY}

Urology and Sexual Medicine guidelines (ISSM, BSSM, AUA, but not European Urology Association [EUA]) tend to refer to "testosterone deficiency syndrome" (TDS), whereas the traditional textbook term "male hypogonadism" is retained in both endocrinology guidelines (ES, AUS). Nevertheless, whether for $\mathrm{MH}$ or TDS, the basic diagnostic criteria across all guidelines are broadly similar, including a combination of clinical symptoms with biochemical evidence of low T, plus an impact on physical health and wellbeing. However, in the interests of consistency, we use MH throughout this review, on the basis that it is an accurate and longstanding medical descriptive term and is consistent with terminology applied across other areas of endocrinology, such as hypo-thyroidism, -parathyroidism, -adrenalism, -pituitarism, etc.

According to ISSM, MH is a clinical and biochemical syn- 
Approach to the man presenting with reduced libido, erectile dysfunction or found to have low testosterone.

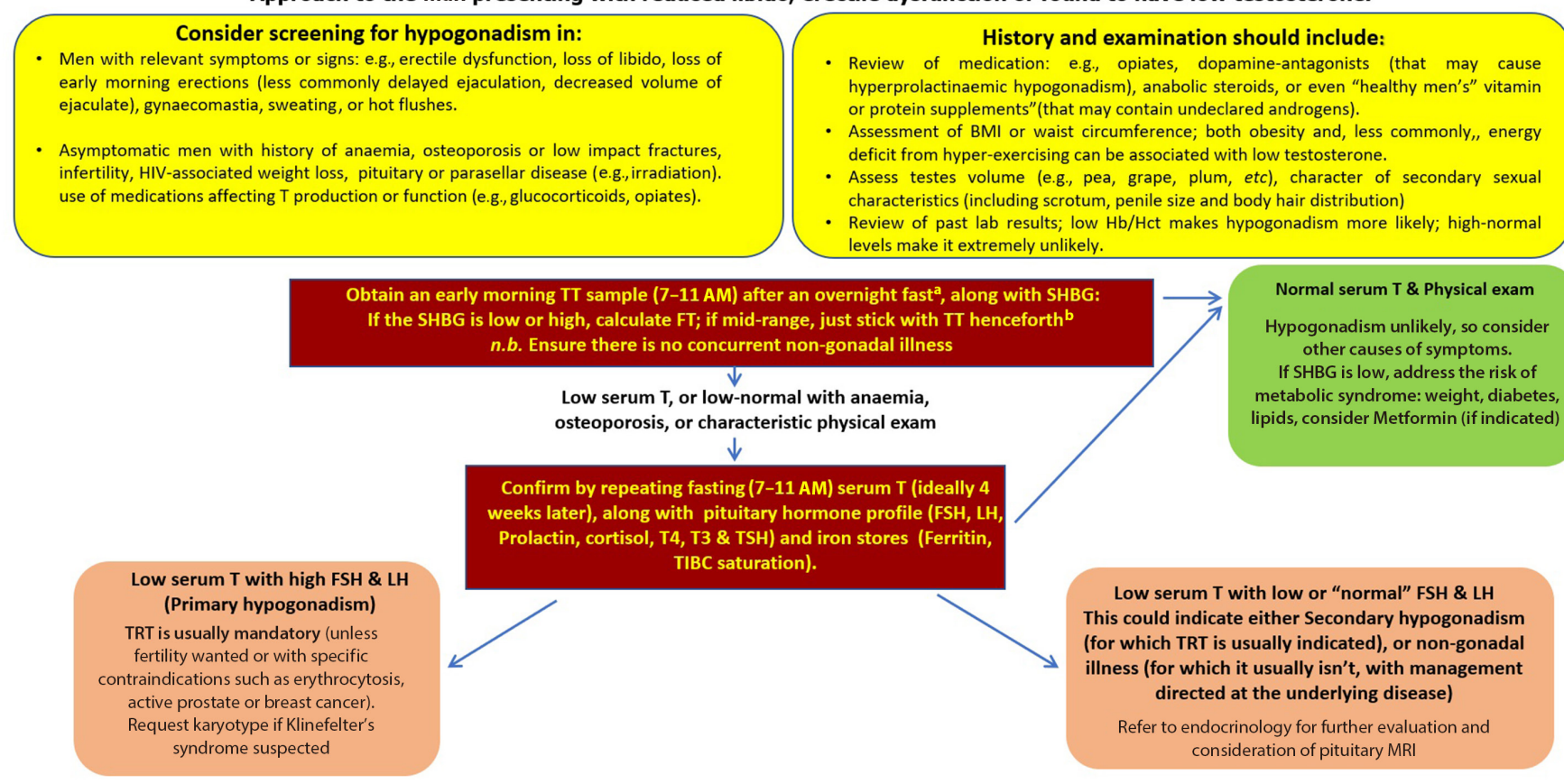

Fig. 2. An approach to low testosterone levels in primary care. HIV, human immunodeficiency virus; BMI, body mass index; Hb/Hct, hemoglobin/hematocrit; TT, total testosterone; SHBG, sex hormone binding globulin; FSH, follicle-stimulating hormone; LH, luteinizing hormone; T4, thyroxine; T3, triiodothyronine; TSH, thyroid stimulating hormone; TIBC, total iron binding capacity; TRT, testosterone replace-

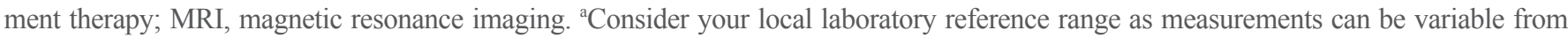
laboratory to another, in shift workers measure within 3 hours of waking; ${ }^{\mathrm{b}}$ www.issam.ch/freetesto.htm.

drome associated with low T (or its action). BSSM and CMAJ concur, but also specifically link $\mathrm{MH}$ to advancing age and accumulating comorbidities, albeit without considering the likelihood of low serum $\mathrm{T}$ in these contexts being instead a non-gonadal epiphenomenon expressed as part of the acute phase response.

The EAU defines $\mathrm{MH}$ as a clinical syndrome caused by androgen deficiency which may adversely affect multiple organ functions and quality of life, and classifies it into four categories: $\mathrm{PH}$, secondary hypogonadism $(\mathrm{HH})$, adult-onset hypogonadism $(\mathrm{AOH})$, and hypogonadism due to defects in androgen target organs. The logic behind the separate AOH category appears initially unclear, in that both $\mathrm{PH}$ and $\mathrm{HH}$ can arise congenitally/in childhood, e.g., Klinefelter (PH) and Kallmann (HH) syndromes, or in adult life; e.g., testicular trauma/torsion/ surgery/orchitis $(\mathrm{PH})$ and prolactinoma $(\mathrm{HH})$. Therefore, creating a non-specific category for $\mathrm{AOH}$ would appear superfluous. However, in practice, this guideline defines $\mathrm{AOH}$ as otherwise unexplained "HH" in obese or older men; i.e., men who might just as easily have functional NGI, and for whom best evidence of benefit lies with addressing underlying general health issues, rather than prescribing $\mathrm{T}[10,13,18,31]$. Regarding the fourth category (defects in androgen target organs), whilst partial and incomplete forms of androgen-insensitivity due to androgen receptor (AR) mutations are increasingly recognized in infertile men [32], evidence for high-dose testosterone being able to surmount these defects is currently slim [33]. There is also an inherent risk of vulnerable normal men being encouraged to seek testosterone treatment in the belief that they may harbor some unspecified resistance to testosterone-action.

The ES defines hypogonadism as a clinical syndrome that results from failure of the testis to produce physiological concentrations of $\mathrm{T}$ and/or a normal number of spermatozoa due to pathology at one or more levels of the hypothalamic-pituitary-testicular axis. The AUS offers a similar definition, and both guidelines exhaustively detail the sub-classification and differential diagnosis of $\mathrm{MH}$.

According to all guidelines, an accurate diagnosis requires the presence of characteristic clinical features and firm biochemical evidence of inappropriately low serum $\mathrm{T}$ level. However, even though it is self-evidently a central clinical manifestation of $\mathrm{MH}$, impaired spermatogenesis only forms part of the diagnostic criteria for ES \& AUS guidance. Providing an ejaculate for 
seminal fluid analysis (SFA) is admittedly expensive, inconvenient and potentially non-specific, in that many men have impaired spermatogenesis unrelated to MH. Nevertheless, if a normal SFA has been demonstrated, then organic MH becomes improbable and, indeed, testosterone treatment will create a risk of infertility that was not previously present. These observations can be deployed to reassure the increasing number of normal men consulting their doctors with TSB.

\section{WHO TO SCREEN FOR MH?}

All major guidelines recommend measuring serum $\mathrm{T}$ in patients who exhibit core clinical features of MH. Although the AUS explicitly advises against screening for hypogonadism in men lacking features suggestive of "pathological hypogonadism," other guidelines endorse a broader screening paradigm, sometimes even in the absence of core symptoms or signs of $\mathrm{MH}$. For instance,

- The CMAJ recommends screening (and potential treatment) of men with otherwise unexplained anemia, sarcopenia, treatment-refractory depression, chronic use of opioids or glucocorticoids, human immunodeficiency virus (HIV)-associated weight loss and erectile dysfunction (ED) resistant to first-line therapy.

- The AUA suggests that measurement of serum T should be considered in men with a history of unexplained anemia, bone density loss, diabetes, exposure of the testes to chemotherapy or ionizing radiation, HIV/acquired immune deficiency syndrome (AIDS), chronic narcotic use, male infertility, pituitary dysfunction, and chronic glucocorticoid use.

- The ISSM recommends screening for MH in men with obesity, T2DM, and metabolic syndrome, while the BSSM recommended screening in men with body mass index $>30 \mathrm{~kg} / \mathrm{m}^{2}$, waist circumference $>102 \mathrm{~cm}$ and T2DM.

- The ES recommends case detection for men deemed to be at increased risk of having hypogonadism and likely to benefit from $\mathrm{T}$ therapy, such as those with low libido, erectile dysfunction, infertility, HIV-associated weight loss, osteoporosis or low-trauma fracture, a history of anabolic steroids use, or using opioids or other drugs or substances that affect $\mathrm{T}$ production or metabolism.

\section{SERUM T CUTOFF TO SUPPORT THE DIAGNOSIS OF MH}

An initial morning measurement of total T concentration is uni- versally recommended for case-detection, but guidelines differ significantly in respect of whether:

- To rely on local laboratory normal ranges, or instead conform to a universal cut-off serum $\mathrm{T}$ value to define the lower limit of normality (LLN); if so, what that value should be?

- Decrementing values for LLN should apply to correspondingly older men?

- Diagnostic venepuncture should always be performed in the fasted state?

- More than one sample is required for diagnostic purposes and, if so, what the minimum spacing between samples should be?

- Serum T values should always be interpreted in the context of patients' clinical presentations?

- Obesity, diabetes, or metabolic syndrome are perceived as increasing the pre-test probability of $\mathrm{MH}$, or conversely, acting to reduce the diagnostic specificity of a low serum $T$ level and increasing the possibility of NGI causing biochemical phenocopy of $\mathrm{HH}$ ?

For instance, the ISSM, BSSM, and EAU recommend diagnosing MH and consider offering TRT on the basis of serum T $<231 \mathrm{ng} / \mathrm{dL}(<8 \mathrm{nmol} / \mathrm{L})$, taken in the morning on two or more separate occasions (spaced at least 4 weeks apart according BSSM, and one week apart according to ISSM). The AUA suggests a higher cutoff of $300 \mathrm{ng} / \mathrm{dL}(<9.2 \mathrm{nmol} / \mathrm{L})$, while the CMAJ does not specify any predetermined serum $\mathrm{T}$ threshold, nor advises any need to repeat sampling if $\mathrm{T}$ was found to be low on the initial measurement.

Only the ES and AUS guidelines specifically recommend that serum $\mathrm{T}$ should always be assessed in context of the patient's clinical presentation, and the result rigorously compared against established levels in healthy eugonadal men in the general population [18]. The ES suggests a threshold of $264 \mathrm{ng} / \mathrm{dL}(9.2$ $\mathrm{nmol} / \mathrm{L}$ ) as LLN for serum $\mathrm{T}$, with its reference range of 9.2 to $31.8 \mathrm{nmol} / \mathrm{L}$ derived from high-order mass spectrometry population from the Centre for Diseases Control (CDC) harmonised standards in healthy non-obese young men [19]. For men having no structural disease of the hypothalamic-pituitary-gonadal (HPG) axis, the AUS has adopted an age-adjusted serum T reference range, e.g., $>10.4 \mathrm{nmol} / \mathrm{L}$ up to age 35 years and $>6.4$ $\mathrm{nmol} / \mathrm{L}$ for men $\geq 70$ years old.

All guidelines agree that the best way to measure $\mathrm{T}$ is by liquid chromatography mass spectrometry, but acknowledge that this may not be available in every institution and; hence, a high-quality validated immunoassay may instead need to be relied upon. 


\section{APPROACH TO LOW-BORDERLINE SERUM T VALUE}

This is the area of greatest variance between different clinical practice guidelines, resulting in markedly heterogenous recommendations. ISSM and BSSM suggest that, in symptomatic men with repeat serum $T$ in the 8 to $12 \mathrm{nmol} / \mathrm{L}$ range, measurement of LH, sex hormone binding globulin (SHBG) and prolactin (PL) should be undertaken, with either high levels of LH (indicating Leydig cell impairment) or SHBG (indicating lower free $\mathrm{T}$ calculated by mass action formula) shifting the equilibrium towards diagnosing $\mathrm{MH}$ and treating with testosterone, but in any case envisioning an individual therapeutic trial of $\mathrm{T}$ (ISSM 6 to 12 months; BSSM 6 months) to determine whether this alleviates symptoms. The CMAJ likewise suggests considering a trial of TRT of 3 months duration in the presence of a convincing clinical picture but uncertain biochemical results. Apart from recommending PL check, neither ISSM, BSSM, nor CMAJ address in any great depth the importance of identifying and treating potential causes of NGI in men with sexual symptoms, low-normal $\mathrm{T}$ and normal $\mathrm{LH}$.

The AUA suggests measuring PL levels in those with repeat low $\mathrm{T}$ and low-normal LH levels; proceeding to treat with $\mathrm{T}$ for $\mathrm{MH}$ if PL levels are normal, and only recommending further endocrine work up and pituitary magnetic resonance imaging if $\mathrm{PL}$ is raised or $\mathrm{T}<150 \mathrm{ng} / \mathrm{dL}$, in order to rule out a structural pituitary lesion.

Free $\mathrm{T}$ calculation by mass action formula was recommended by the BSSM, ISSM EUA, and ES. The EAU recommends calculating free $\mathrm{T}$ in case of discrepancy between serum (total) $\mathrm{T}$ and symptoms, while the ES recommends the use of calculated free $\mathrm{T}$ in the presence of conditions that alter SHBG levels, or when serum $\mathrm{T}$ levels are in the borderline range. By contrast, the AUS is broadly opposed to using calculated free $\mathrm{T}$ to inform clinical decision-making, due to the highly uncertain correlation between values derived from any of the published formulae and actual laboratory measurements by equilibrium dialysis [34].

Nevertheless, a pragmatic approach to evaluating calculated free $\mathrm{T}$ would acknowledge that, whilst it is unlikely to provide an accurate measurement of actual circulating unbound $\mathrm{T}$, studies have found it to correlate better than total $\mathrm{T}$ with several biological read-outs of androgen action, including bone density [35], sexual function [36], and haematopoeisis [37]. However, where SHBG level is mid-range, the calculation adds little added value [21].

\section{INDICATIONS FOR TESTOSTERONE TREATMENT}

All guidelines affirm that testosterone replacement is indicated with a verified diagnosis of $\mathrm{MH}$, i.e., the presence of characteristic symptoms combined with the unequivocal biochemical finding of low T. However, as discussed above, some guidelines (particularly ISSM, BSSM, and CMAJ) apply significantly lower thresholds for treatment, and endorse trials of testosterone treatment with symptoms conceivably due to $\mathrm{MH}$, even when biochemistry is not entirely supportive, or potentially due to NGI.

Surprisingly little weight is given by any guideline to the key significance of a raised LH level - a finding that not only highlights an unequivocal defect of Leydig cell function, but also usefully excludes physiological NGI and venepuncture mistiming as a cause of lower serum $\mathrm{T}$ - or of a high-normal hemoglobin or hematocrit (Hct), which indicates that testosterone treatment will necessarily carry an unacceptably high risk of inducing erythrocytosis [21].

According to ES and AUS, hypogonadal men with a disease affecting the testis $(\mathrm{PH})$ or hypothalamo-pituitary-testicular (HPT) axis (HH) are classified as having organic/classical (ES) or pathological (AUS) MH, with testosterone treatment being generally indicated. AUS in particular emphasises the importance of being able to frame a diagnosis of $\mathrm{MH}$ in the context of a recognised cause, i.e., recognition of $\mathrm{MH}$ is not the final diagnosis in itself, but rather the entry-point to achieving an overarching diagnosis.

In contrast testosterone treatment is not generally recommended by ES or AUS for the other category of men having intrinsically intact HPT axis, but suppressed function due to other causes (e.g., obesity or other comorbidity). ES defines these men as having functional $\mathrm{MH}$, whereas AUS does not consider them as having any form of MH. We favour the term NGI, first coined by Gan et al. [8], reflecting analogous physiological significance to non-thyroidal illness syndrome. Both ES and AUS emphasise the primacy of treating underlying health problems in these men; an approach that can reverse functional HH/NGI biochemistry. In contrast, other guidelines do not differentiate between organic/classical/pathological $\mathrm{MH}$ and functional $\mathrm{HH} /$ NGI in terms of indications for treatment, and are also generally content to define MH (or TDS) as a top-level hierarchical diagnosis. 


\section{AGING, OBESITY, AND OTHER COMORBIDITIES}

Clinical practice guidelines generally acknowledge that the prevalence of subnormal serum $\mathrm{T}$ levels increases with advancing age; potentially resulting either from $\mathrm{PH}$ (due to age- or comorbidity-related related decline in Leydig cell function) or from HH/NGI (due to age-related accumulation of comorbidities). The ES considers advanced age to be a cause of organic $\mathrm{PH}$, but not a cause of organic $\mathrm{HH}$, whereas AUS does not consider age to cause any form of $\mathrm{MH}$ and does not necessarily consider a raised LH level in an older man as indicating pathological MH meritorious of testosterone treatment.

Whilst acknowledging that aging men are prone to accumulating comorbid illnesses resulting in low serum $T$ levels, the ISSM, and BSSM nevertheless define this as a form of age-related $\mathrm{MH}$, whose treatment implications are not substantially different from those of classical or organic $\mathrm{MH}$. The CMAJ, ISSM, and BSSM state that obesity, T2DM, and metabolic syndrome are all strongly associated with MH. Indeed, these three guidelines are heavily influenced by indirect and/or observational data supporting the "Cohen hypothesis" of a bi-directional relationship between serum $\mathrm{T}$ and fat mass, along with a role for $\mathrm{T}$ therapy in helping to mitigate these interlinked non-gonadal conditions through promotion of muscle anabolism, lipolysis and weight-loss [38]. Consequently, they recommend systematic screening for $\mathrm{MH}$ in those clinical settings, even in the absence of symptoms or signs, which is a radical and potentially resource-intense departure from traditional case-finding practice.

Hence, the ISSM endorses testosterone treatment in men with low $\mathrm{T}$ associated with obesity and metabolic syndrome, particularly if the clinician suspects that the patient will not adequately adhere to the recommended dietary, behavioural (e.g., abstain from opiates) and exercise regimen. Similarly, the BSSM endorses lifestyle modification in addition to testosterone treatment in men with HH/NGI due to obesity who have distressing symptoms. The AUA also recommends consideration of screening for $\mathrm{MH}$ in patients with diabetes, without specifying which type of diabetes, and - beyond initiation of testosterone treatment-makes no recommendations on how to approach men with $\mathrm{HH} / \mathrm{NGI}$ and metabolic health issues, apart from recommending referral to Endocrinology if PL is found to be raised.

Interestingly, recent data from the T4DM trial [39] have shown that adding testosterone treatment to lifestyle modifications in nearly 1,000 obese men was associated with a markedly reduced prevalence of T2DM at 2 years $(12.4 \%$ vs. $21.1 \%$, pla- cebo) and also had small favourable outcome on sexual function. However, it led to a dangerously high Hct ( $>54 \%)$ developing in $21.6 \%$ of subjects, compared with only $1.2 \%$ in the placebo arm. Moreover, men in the $\mathrm{T}$ group reported more adverse events (e.g., ischaemic heart disease, arrhythmias, benign prostate enlargement), 55 compared with 42 events in the placebo group. Death rates were identical, two in each group, although the study was not powered to evaluate this.

By contrast, the EUA, ES, and AUS all recommend lifestyle modification and addressing the underlying comorbid illness as a first line intervention, with the EUA also recommending the use of PDE5-inhibitors, as a first line therapy ahead of testosterone for erectile dysfunction, in symptomatic men with low serum $\mathrm{T}$. The ES and AUS concur that functional $\mathrm{HH} / \mathrm{NGI}$ due to obesity can be reversed by addressing the excess weight through diet and exercise. Having been drafted prior to publication of "The T Trials" (TTT) [40], the AUS also noted only limited data from high-quality randomised controlled trials of testosterone, achieving clinically significant outcomes in older men - usually with chronic disease (such as obesity) — having low T levels but no evidence of pathological MH. Fundamentally, the AUS considers low $\mathrm{T}$ in this context to be marker of the underlying poor health, and hence does not support prescribing of testosterone in such settings. Detailed analysis of TTT is beyond the scope of this review, but their findings can be summarised as follows in respect of older men with obesity and metabolic syndrome:

- Testosterone was marginally more effective than PDE5-inhibitors in respect of sexual functioning; albeit any superiority may not last beyond a year from initiation.

- Testosterone did not improve vitality score, physical function, or cognitive function.

- Testosterone increased coronary artery non-calcified plaque volume as assessed using computed tomographic angiography, indicating potential adverse $\mathrm{CV}$ risk.

- Testosterone increased hemoglobin in both men with anemia of a known cause and in those having unexplained anemia.

- Testosterone increased volumetric bone mineral density and the estimated bone strength of the spine and hip.

Informed by TTT data, current ES guidance is significantly more stringent in respect of the diagnosis of $\mathrm{MH}$ and eligibility for testosterone treatment compared with its previous 2010 iteration [41] and is thus much closer to AUS. Nevertheless, for reasons that are not explored in great detail, but may relate to not wishing to alienate certain elements of their membership, the ES continues to allow clinicians to "individualize the decision to treat or not to treat with $\mathrm{T}$ based on careful consideration of the 
severity of symptoms, the degree of T deficiency, confounding influence of the comorbid illness, patient preferences, and the uncertainty of the risks and benefits of testosterone therapy." In our view, this caveat is inconsistent with the overall direction of the updated ES guidance, in that it leaves open the possibility for prescribing $\mathrm{T}$ based solely upon patient expectations.

\section{CAUSES OF HH THAT SHOULD NOT BE MISSED?}

That HH (whether organic or functional) is not necessarily a final diagnosis, but potentially the first manifestation of another disease, is regrettably overlooked by some guidelines. Most guidelines agreed on the need of measuring serum LH (to differentiate PH from $\mathrm{HH}$ ) and PL levels (to assess whether a pituitary adenoma is likely to be present). However, the AUA and BSSM recommended pituitary imaging only if PL is persistently elevated and/or in the presence of severe biochemical T deficiency $(<5.2 \mathrm{nmol} / \mathrm{L},<150 \mathrm{ng} / \mathrm{dL})$. This might not be the safest approach, given that structural pituitary abnormalities are more common in isolated $\mathrm{HH}$ than in the general population and total T might not be always below the aforementioned threshold [42]. Indeed, such recommendation might underestimate the presence of a non-functioning pituitary tumour (or a diagnosis of pan-hypopituitarism) manifesting as mild- moderate $\mathrm{HH}$ with low-normal PL levels (which is not an uncommon presentation to endocrinology services). Although ES guidelines quote the same threshold, they do so on the basis of significantly more detailed clinical context: "Clinicians can improve the diagnostic yield of pituitary imaging to exclude pituitary and/or hypothalamic tumors by performing this procedure in men with panhypopituitarism, persistent hyperprolactinemia, serum TT $150 \mathrm{ng} / \mathrm{dL}(5.2$ $\mathrm{nmol} / \mathrm{L}$ ), or symptoms of tumor mass effect (e.g., visual impairment, visual field defect, or new-onset headache)."

In addition to pituitary disease, investigating other potential causes of $\mathrm{HH}$ is recommended by some, but not all guidelines. Hereditary hemochromatosis (HHem), for example, is a genetic disease that promotes tissue iron overload that may lead to $\mathrm{HH}$ through gonadotroph iron toxicity, along with potentially serious cardiac, hepatic and joint disease, and diabetes [43]. Diabetes occurs in around $50 \%$ of patients with $\mathrm{HHem}$, with $\mathrm{MH}$ (mostly SH) being the second most prevalent endocrinopathy after diabetes [44]. More importantly, recovery of reproductive function and reversibility of $\mathrm{HH}$ have been reported after aggressive venesection therapy $[45,46]$. Disappointingly, only CMAJ, ES, and AUS clearly indicate the need to measure se- rum ferritin or transferrin saturation (both very simple and cheap tests) in men with SH.

The ISSM inaccurately defines HHem solely as a cause of $\mathrm{PH}$, whereas a largely pituitary-level defect $(\mathrm{HH})$ in HHem is indicated by the literature $[43,47]$. The BSSM, AUA, EUA, made no mention of HHem or the need to screen for it, which is very surprising given their emphasis on screening for $\mathrm{MH}$ in patients with T2DM.

Most guidelines acknowledge that $\mathrm{HH}$ can arise due to legitimate or illegitimate use of prescription medications (e.g., opiates and androgens), but ISSM, BSSM, AUA, EUA, and CMAJ provide no guidance on how best to approach men with $\mathrm{HH}$ in such settings. The ES sensibly recommends only considering $\mathrm{T}$ treatment of opiate-induced hypogonadism in men with distressing symptoms and in whom opiate-withdrawal is not an option. The AUS stresses that opiates or androgens cause functional and reversible $\mathrm{HH}$ and that cessation of the offending drug should always be attempted first. If this is not possible then an endocrinology referral is warranted to discuss the risks and benefits of any suggested intervention.

\section{TESTOSTERONE TREATMENT AND ANDROGEN-SENSITIVE CANCERS}

Most guidelines counsel against testosterone in patients with metastatic or locally advanced prostate cancer (PCa), any form of male breast cancer, and in patients at high risk for recurrent PCa. The ISSM and AUA recommend a mutual decision to be made between the patient and the physician taking into account benefits versus the potential risks. The BSSM, EAU, and CMAJ recommended offering testosterone to symptomatic men with history of treated localized low-risk PCa (Gleason score $<8$, stages $1-2$, preoperative prostate specific antigen [PSA] levels $<10 \mathrm{ng} / \mathrm{mL}$, and not within 1 year of treatment with curative intent) and without evidence of active disease (based on measurable PSA level, digital rectal examination [DRE] result, and imaging evidence of metastatic disease). The EAU advise that testosterone can be cautiously offered to patients who underwent brachytherapy or external beam radiation for low-risk prostate cancer.

The ES advises that all men receiving testosterone treatment should have urological evaluation upon the detection of prostate nodule or induration, or achieving $\geq 40 \%$ rise in PSA level over the first year of treatment. ES advises against testosterone in men with prostate cancer, palpable prostate nodule or induration, PSA level $\geq 4 \mathrm{ng} / \mathrm{mL}$ (or $\geq 3 \mathrm{ng} / \mathrm{mL}$ if combined with a 
high risk of prostate cancer such as African-American origin and those with first degree relatives with having prostate cancer) without urological evaluation. In relatively younger men (age 55 to 69) with life expectancy of $\geq 10$ years or men aged 40 to 69 who have high risk (African-American origin and those with first degree relatives with having prostate cancer), the ES suggests shared decision making after discussing risks and benefits, and potential prostate monitoring. Eugonadal men who develop androgen-sensitive prostate cancer, treated with curative intent, routinely receive androgen-deprivation therapy for a period of 6 to 24 months (depending on tumor phenotype) [48] and, therefore, from basic first principles, the same occurrence in a hypogonadal man ought not prompt interruption of testosterone treatment for longer than 2 years.

All guidelines emphasise precautionary baseline PSA measurement and DRE, with periodic repeats during treatment. However, in practice, Endocrinologists tend not to perform DRE; instead relying on biochemical PSA monitoring, which - apart from rare cases of non-secretory $\mathrm{PCa}$ - is in any case far more sensitive. It may be that urologists recommend DRE mainly because it is part of their standard clinical examination, and that endocrinologists lack the necessary confidence and experience in the area to offer a disenting opinion. This is indeed an area that is ripe for a truly multidisciplinary review of the evidence.

Indeed, among men with congenital $\mathrm{MH}$, the occurrence of PCa during $\mathrm{T}$ treatment is so vanishingly rare that we have been able to identify less than 10 published reports [49].

\section{TESTOSTERONE AND CV DISEASE}

Current guidelines state that there is no credible evidence that testosterone increases the risk of $\mathrm{CV}$ events, provided that it is prescribed appropriately to men with a well-founded diagnosis of MH. Nevertheless, two landmark clinical trials raised CV safety concerns when testosterone was prescribed to older men having a primary diagnosis of age-related frailty [50], or having HH/NGI associated with obesity, T2DM or metabolic syndrome [40]. Moreover, data from the Copenhagen General Population Study found a $50 \%$ increased risk of CV thrombosis among men in the top 5\% for Hct $(0.48 \%)$, compared with the 25 th to 50 th centiles [51]; thereby indicating a mechanism by which testosterone (or indeed smoking and chronic lung disease) might plausibly increase $\mathrm{CV}$ risk. It may be that a higher Hct directly promotes thrombogenicity; alternatively, secondary, or relative polycythaemia (where red cell mass is normal, but plasma volume is reduced) may simply be a manifestation of the in- creased vascular tone associated with cardiometabolic disease [52]. Finally, no study has properly considered testosterone's action in shortening the cardiac QT interval as a potential contributing factor to $\mathrm{CV}$ risk; by contrast, a possible role for testosterone to treat men predisposed to short-QT-arrhythmias is currently being investigated [53].

The ISSM recommends that MH in men with $\mathrm{CV}$ disease be assessed and monitored in the same way as in other men, whereas the BSSM recommends assessing CV risk factors and optimising secondary prevention in men with established disease before commencing testosterone. The EUA suggests a greater need for caution in men with pre-existing CV disease and, potentially, considering echocardiography before initiation of testosterone. The AUA recommended the need to counsel patients prior to starting testosterone, explaining that the evidence is patchy and that it is unknown whether testosterone can increase or decrease the risk of any major adverse CV event. The CMAJ guideline makes a weak recommendation, based on low-quality evidence, that testosterone treatment in men with $\mathrm{CV}$ disease be restricted to those with stable disease and only after a discussion of the potential risks and benefits. More coherently, both AUA and ES caution against initiating $\mathrm{T}$ with recent $\mathrm{CV}$ or stroke event ( 3 to 6 months for the AUA and 6 months for the ES) while the AUS suggestes the need for caution when using $T$ in older men with known CV disease.

The UK Society for Endocrinology has produced a brief position statement on testosterone in older men [54]. Whilst recognizing the uncertainty and potential risks around the prescribing of $\mathrm{T}$ in obese and/or older men with low $\mathrm{T}$, it notes that the overwhelming majority of older men retain Leydig cell function into old age and that, consequently, there is no logical underpinning for withholding testosterone treatment in symptomatic older men with a verified diagnosis of organic or syndromic $\mathrm{MH}$.

\section{MONITORING TESTOSTERONE THERAPY}

To avoid testosterone-induced erythrocytosis, many guidelines counsel against initiating $\mathrm{T}$ in patients with baseline elevated Hct, which makes sense in terms of mitigating CV risk. However, surprisingly underplayed in all guidelines, is the concept that a raised Hct makes $\mathrm{MH}$ rather improbable in the first place.

However, where threshold Hct values - beyond which testosterone treatment is inadvisable are quoted in some guidelinesthese are surprisingly high, $>48 \%$ according to the ES and (amazingly) $>54 \%$ according to the BSSM and EUA. Not only would baseline Hct $>50 \%$ tend to refute the diagnosis of $\mathrm{MH}$, 
but initiation of testosterone therapy would be predicted to result in unacceptable erythrocytosis with near certainty. Both ES and AUS recommend monitoring Hct after 3 to 6 months of testosterone initiation and annually thereafter; sensibly recommending that the testosterone dose be reduced should erthrocytosis begin to develop. However, BSSM instead suggests periodic venesection, whilst potentially continuing the existing dose of testosterone; a practice common to athletes abusing androgens and for which safety and efficacy data are notably lacking.

The ISSM recommends targeting $\mathrm{T}$ levels at mid-point of the reference range, with bloods taken at each follow-up visit, with the initial monitoring biochemistry varied according to the preparation and route of administration. The BSSM recommends a therapeutic target of mid-upper-end of range (15 to 30 nnmol/L), with blood tests (T, SHBG, and albumin) checked at least 1 to 2 hours after topical testosterone applications, but states that "timing for blood testing is irrelevant" in relation to injectable testosterone. The AUA recommends using the lowest possible dose of testosterone to achieve levels of 450 to $600 \mathrm{ng} /$ dL ( 15.6 to $20.8 \mathrm{nmol} / \mathrm{L}$ ) and, for men on long-acting injectables, to have blood works done midway between injections. The CMAJ recommends a therapeutic target of 14.0 to 17.5 $\mathrm{nmol} / \mathrm{L}$, but makes no recommendations on timing and intervals of monitoring. The EUA acknowledges a lack of data to define an optimal therapeutic target, but their expert consensus suggests aiming for the mid-normal age-adjusted range, or whatever level alleviates symptoms. For men receiving injectable testosterone, having a significant peak-trough difference in levels, the ES recommends monitoring blood tests be performed done at the mid-point between injections.

Nevertheless, in the UK healthcare environment, doing monitoring blood tests immediately pre-injection at "trough" and aiming for low-normal serum $\mathrm{T}$ levels achieves greater convenience and simplicity for both patients and clinicians [13]. Moreover, whereas almost all guidelines recommend aiming for a midrange serum $\mathrm{T}$ level, they universally fail to explicitly articulate the importance of qualifying this advice with the necessary contextual prefix "other things being equal." Thus, for men predisposed to developing erythrocytosis, it may prove necessary to accept lower serum $\mathrm{T}$ in order to mitigate risks of thrombosis or venesection. In contrast, for younger men with anemia, or low bone density, achieving a higher level is probably justified [21].

\section{DISCUSSION}

The aim of clinical practice guidelines is to analyse complex or incomplete evidence and provide clear guidance to practicing physicians. However, a distinct lack of consensus on how to approach $\mathrm{MH}$ is evident from the guidelines that we reviewed, hence potentially adding to confusion and uncertainty in certain areas, rather than reducing it.

From the epidemiological perspective, there is increasing concern that "the system is broken" in that large numbers of men with organic $\mathrm{MH}$, who would certainly benefit from testosterone treatment, are not currently being identified by modern medical practice [54]. Conversely, even larger numbers of men have been prescribed testosterone without no greater diagnostic granularity that an isolated random low serum $\mathrm{T}$ level, or even a low score on an "androgen deficiency" questionnaire, with no biochemical workup $[3,18]$.

Some guidelines accept the routine diagnosis of $\mathrm{HH}$ in men with borderline-low T and low-normal LH associated with comorbidities - consistent with NGI — and also tolerate androgeninduced polycythemia up to a remarkably unphysiological $54 \%$ Hct [55]. Moreover, use of the term TDS, rather than MH, may unnecessarily imply a need to replace testosterone in a man whose HPT axis is fundamentally intact.

This situation is unlikely to improve as long as guidelines continue to display markedly heterogenous recommendations in major area of clinical practice, including:

- Basic terminology: whether to remain with $\mathrm{MH}$, or move to a new descriptor, TDS? We suggest sticking with MH.

- Is MH (or TDS) a final diagnosis in its own right, or does it require a higher-order descriptor in order to be fully characterized and credible, such as $\mathrm{HH}$ due to opiate use, or PH due to Klinefelter syndrome? We suggest the latter.

- HPG axis suppression due to co-morbidities such as obesity: should it be considered a form of HH like any other, and treated accordingly with testosterone; a form of functional, or nonorganic $\mathrm{HH}$, not usually treated with $\mathrm{T}$, or is it instead better described as physiological NGI-effect for which a logical basis for testosterone prescribing, along with data on risks and benefits, are all notably lacking? We suggest the latter.

- Case-finding paradigms: apart from men with core clinical features or risk factors (sexual dysfunction, infertility, gynecomastia, vasomotor symptoms, anemia, osteoporosis, absent or incomplete secondary sexual characteristic, characteristic syndromic features, or history of tumor, surgery, chemotherapy, or ionizing radiation affecting the HPT axis), who else - if anybody — should be screened for MH?

- Specifically, should men with ongoing opiate use, prior nonprescription androgen use, or obesity/metabolic syndrome be 
Table 1. Clinical Practice Guidelines and Their Cutoff for Serum TT

\begin{tabular}{|c|c|c|}
\hline Guideline & T cutoff, $\mathrm{nmol} / \mathrm{L}$ & Evidence for the cut off \\
\hline ISSM, BSSM & $<8$ & $\begin{array}{l}\text { Cross-sectional cohort study of } 434 \text { men (age } 50-86 \text { years) which showed that ED was more prevalent at this TT } \\
\text { level [57] }\end{array}$ \\
\hline AUA & $<10.4$ & $\begin{array}{l}\text { Meta-analysis of RCTs including studies where TT }<350 \mathrm{ng} / \mathrm{dL} \text {, with a median baseline TT of } 249 \mathrm{ng} / \mathrm{dL} \text { and an } \\
\text { interquartile range of } 233-283 \mathrm{ng} / \mathrm{dL}[27,58]\end{array}$ \\
\hline EUA & $<8$ & A survey of 3,369 middle aged men (age 40-79 years) [59] \\
\hline ES & $<9.2$ & $\begin{array}{l}\text { Data from harmonized reference range from } 100 \text { healthy young nonobese men from } 4 \text { cohorts: the Framingham } \\
\text { Heart Study, European Male Aging Study, Osteoporotic Fractures in Men Study, and Male Sibling Study of } \\
\text { Osteoporosis [60] }\end{array}$ \\
\hline
\end{tabular}

TT, total testosterone; ISSM, International Society of Sexual Medicine; BSSM, British Society of Sexual Medicine; ED, erectile dysfunction; AUA, American Urological Association; RCT, randomized clinical trials; EUA, European Urology Association; ES, Endocrine Society.

screened for $\mathrm{MH}$, even in the absence of relevant symptoms or signs, or is evidence of treatment-safety or -benefit inadequate for these? All but the AUS endorse testosterone prescribing for medically-justified opiate use $[29,56]$.

- Presence of obesity/metabolic syndrome: is this a valid justification for actively seeking-out $\mathrm{MH}$, or instead, a reason to be very cautious in interpreting low serum $\mathrm{T}$ levels. We suggest the latter.

- Diagnostic threshold: what should the serum T cut-off be for the diagnosis of MH? Among the clinical practice guidelines, the cut-off was variable and sometimes driven by studies looking mainly at certain symptoms national (Table 1) [27,57$60]$. We suggest that individual laboratories engage with quality-control measures at a national level, aiming to achieve standardized reference ranges, rather than for guideline-writers to impose arbitrary thresholds.

- Interpretation of a borderline T level: should age-adjusted ranges be adopted, or should the normal range derive from healthy younger men? We can see the merits of both approaches; the important thing is for the derivation of the reference range (healthy men or "all-comers"; age-adjusted or not?) to be clearly indicated on laboratory reports, as this will necessarily affect their interpretation by clinicians.

- To what extent should levels of LH or SHBG influence the interpretation of serum total $\mathrm{T}$; do free $\mathrm{T}$ calculations accurately correlate with values directly measured by equilibrium dialysis and does either measurement add extra diagnostic value? We believe that a consistently raised LH level unequivocally indicates Leydig cell insufficiency and, if associated with anemia, low bone density, painful gynecomastia, or treatment- refractory sexual dysfunction, merits consideration of testosterone treatment even when serum $\mathrm{T}$ is in the lower quartile, rather than being frankly below range. We also believe calcu- lated free-T to be a useful adjunct to clinical decision-making when the SHBG level is an "outlier," although we do not necessarily consider it to accurately reflect biochemical reality at tissue level.

- Hct: what is the threshold value above which testosterone treatment is contraindicated and what is the maximum tolerated value on treatment and is venesection a safe and reasonable alternative to testosterone dose-reduction? We do not generally consider initiating testosterone treatment in men with Hct $\geq 48 \%$, and would always titrate treatment so as to maintain it $\leq 50 \%$, even if this means accepting a lower serum $\mathrm{T}$ value. This strategy has enabled us to avoid referring patients for venesection.

- CV disease: do concerns only apply to men with frailty- or obesity-associated HH/NGI treated with testosterone, or also to men with organic, syndromic, or pathological MH? We strongly believe the former to be the case.

- Prostate safety: is there any value in monitoring prostate safety for men having a verified diagnosis of $\mathrm{MH}$ and treated with testosterone, above any beyond the recommended level of screening in the background male population, and if so, does performing DRE offer any greater benefit than measurement of serum PSA level? Although firmly believing that greater interdisciplinary consensus is required in this area, we do not routinely perform DRE in our patients, but we do monitor PSA.

- Old age: with increasing numbers of men surviving into their 9th and 10th decades, is there any chronological age above which concerns arise in respect of testosterone prescribing, even with a verified diagnosis of organic, syndromic, or pathological MH? In the absence of a ubiquitous male "andropause," and with the overwhelming majority of older men broadly maintaining Leydig cell sensitivity to LH, we can en- 
visage no upper age limit for prescribing testosterone when MH has been accurately diagnosed.

- T2DM: given the strong association with PH, is there a causal relationship between T2DM and $\mathrm{PH}$ and what is the direction of causation? Unfortunately, research in the area of MH and T2DM has overwhelmingly focused on $\mathrm{HH} / \mathrm{NGI}$, rather than $\mathrm{PH}$.

Finally, it is estimated that patients with chronic diseases end up delivering the major proportion of their overall medical care, by keeping track of and attending appointments, attending for monitoring bloods, requesting and collecting drug prescriptions, reporting clinical responses and adverse effects, and adhering to lifestyle guidance. For many other chronic diseases, patients are increasingly contributing to guidance, but with notable exceptions [61] the patient experience of treatment in $\mathrm{MH}$ - frequently preceded by a prolonged diagnostic odyssey - is not wellrepresented in guidance.

\section{CONCLUSIONS}

Current national and international guidelines relating to $\mathrm{MH}$ are remarkably heterogeneous in respect of diagnostic, treatment and monitoring criteria. Indeed, several guidelines do not even refer to $\mathrm{MH}$ as such, but rather to "testosterone deficiency." Specifically, for men having low serum $\mathrm{T}$ associated with low or "inappropriately normal" gonadotropins (a biochemical fingerprint that is shared by both $\mathrm{HH}$ and NGI) guidelines vary hugely in the degree to which further evaluation for underlying local or systemic co-morbidities in indicated in order to identify a proximate cause or overarching diagnosis.

There are also major variances as to whether "functional HH" (or NGI) associated with obesity and metabolic syndrome should be considered for testosterone therapy, whether as a firstline treatment, second-line after lifestyle change, or not at all. Crucially, although testosterone ameliorates some surrogate markers of $\mathrm{CV}$ risk such as lean body mass and biochemical metabolic profiles and also improves bone density and hematocrit, it does not greatly improve sexual function, physical or mental vitality. Indeed, there are persisting concerns relating to $\mathrm{CV}$ disease that may relate to a counterbalancing risk arising from greater propensity to arterial thrombosis with rising hematocrit.

At the same time, many men with organic $\mathrm{MH}$, who would be expected to benefit from testosterone treatment, continue to remain undiagnosed and untreated due to deficient basic and postgraduate medical training in sexual medicine. Indeed, the voices and perspective of patients affected by $\mathrm{MH}$ have been conspicu- ously lacking in relation to the process of drafting guidance.

We hope this review will allow clinicians to navigate the field of $\mathrm{MH}$ with greater confidence and, thereby, offer the most appropriate and safe advice and management subject to limitations of the existing evidence base.

\section{CONFLICTS OF INTEREST}

No potential conflict of interest relevant to this article was reported.

\section{ORCID}

Ahmed Al-Sharefi https://orcid.org/0000-0002-9777-5317

Richard Quinton https://orcid.org/0000-0002-4842-8095

\section{REFERENCES}

1. Bhasin S, Brito JP, Cunningham GR, Hayes FJ, Hodis HN, Matsumoto AM, et al. Testosterone therapy in men with hypogonadism: an Endocrine Society clinical practice guideline. J Clin Endocrinol Metab 2018;103:1715-44.

2. Harman SM, Metter EJ, Tobin JD, Pearson J, Blackman MR; Baltimore Longitudinal Study of Aging. Longitudinal effects of aging on serum total and free testosterone levels in healthy men. Baltimore Longitudinal Study of Aging. J Clin Endocrinol Metab 2001;86:724-31.

3. Araujo AB, Esche GR, Kupelian V, O’Donnell AB, Travison TG, Williams RE, et al. Prevalence of symptomatic androgen deficiency in men. J Clin Endocrinol Metab 2007;92:4241-7.

4. Llahana S, Follin C, Yedinak C, Grossman AB. Advanced practice in endocrinology nursing. Cham: Springer; 2019. Chapter, Classification of hypothalamic-pituitary-gonadal (HPG) axis endocrine disorders; p. 853-70.

5. Kelly DM, Jones TH. Testosterone and obesity. Obes Rev 2015;16:581-606.

6. Pye SR, Huhtaniemi IT, Finn JD, Lee DM, O’Neill TW, Tajar A, et al. Late-onset hypogonadism and mortality in aging men. J Clin Endocrinol Metab 2014;99:1357-66.

7. Dong JY, Zhang YH, Qin LQ. Erectile dysfunction and risk of cardiovascular disease: meta-analysis of prospective cohort studies. J Am Coll Cardiol 2011;58:1378-85.

8. Wu FC, Tajar A, Pye SR, Silman AJ, Finn JD, O’Neill TW, et al. Hypothalamic-pituitary-testicular axis disruptions in older men are differentially linked to age and modifiable risk factors: the European Male Aging Study. J Clin Endo- 
crinol Metab 2008;93:2737-45.

9. Turner HE, Wass JA. Gonadal function in men with chronic illness. Clin Endocrinol (Oxf) 1997;47:379-403.

10. Dwyer AA, Quinton R. The metabolic syndrome in central hypogonadotrophic hypogonadism. Front Horm Res 2018; 49:156-69.

11. Cheng J, Han B, Li Q, Xia F, Zhai H, Wang N, et al. Testosterone: relationships with metabolic disorders in men-an observational study from SPECT-China. Int J Endocrinol 2017; 2017:4547658.

12. Sartorius G, Spasevska S, Idan A, Turner L, Forbes E, Zamojska A, et al. Serum testosterone, dihydrotestosterone and estradiol concentrations in older men self-reporting very good health: the healthy man study. Clin Endocrinol (Oxf) 2012;77:755-63.

13. Society for Endocrinology. Society for endocrinology position statement on male hypogonadism and ageing [Internet]. Bristol: Society for Endocrinology; 2018 [cited 2020 Aug 4]. Available from: https://www.endocrinology.org/media/2710/ male-hypogonadism-and-ageing-2018.pdf.

14. Bojesen A, Kristensen K, Birkebaek NH, Fedder J, Mosekilde $\mathrm{L}$, Bennett $\mathrm{P}$, et al. The metabolic syndrome is frequent in Klinefelter's syndrome and is associated with abdominal obesity and hypogonadism. Diabetes Care 2006;29:1591-8.

15. Dahlqvist JR, Vissing J. Diabetes in myotonic dystrophy. Front Diabetes 2017;25:182-7.

16. Baillargeon J, Urban RJ, Ottenbacher KJ, Pierson KS, Goodwin JS. Trends in androgen prescribing in the United States, 2001 to 2011. JAMA Intern Med 2013;173:1465-6.

17. Layton JB, Li D, Meier CR, Sharpless JL, Sturmer T, Jick $\mathrm{SS}$, et al. Testosterone lab testing and initiation in the United Kingdom and the United States, 2000 to 2011. J Clin Endocrinol Metab 2014;99:835-42.

18. Gan EH, Pattman S, Pearce SHS, Quinton R. A UK epidemic of testosterone prescribing, 2001-2010. Clin Endocrinol (Oxf) 2013;79:564-70.

19. Handelsman DJ. Trends and regional differences in testosterone prescribing in Australia, 1991-2001. Med J Aust 2004; 181:419-22.

20. Karavolos S, Reynolds M, Panagiotopoulou N, McEleny K, Scally M, Quinton R. Male central hypogonadism secondary to exogenous androgens: a review of the drugs and protocols highlighted by the online community of users for prevention and/or mitigation of adverse effects. Clin Endocrinol (Oxf) 2015;82:624-32.

21. Al-Sharefi A, Wilkes S, Jayasena CN, Quinton R. How to

538 www.e-enm.org manage low testosterone level in men: a guide for primary care. Br J Gen Pract 2020;70:364-5.

22. Calof OM, Singh AB, Lee ML, Kenny AM, Urban RJ, Tenover JL, et al. Adverse events associated with testosterone replacement in middle-aged and older men: a metaanalysis of randomized, placebo-controlled trials. J Gerontol A Biol Sci Med Sci 2005;60:1451-7.

23. Budoff MJ, Ellenberg SS, Lewis CE, Mohler ER 3rd, Wenger NK, Bhasin S, et al. Testosterone treatment and coronary artery plaque volume in older men with low testosterone. JAMA 2017;317:708-16.

24. Dean JD, McMahon CG, Guay AT, Morgentaler A, Althof $\mathrm{SE}$, Becher EF, et al. The International Society for Sexual Medicine's process of care for the assessment and management of testosterone deficiency in adult men. J Sex Med 2015;12:1660-86.

25. Hackett G, Kirby M, Edwards D, Jones TH, Wylie K, OsseiGerning N, et al. British Society for Sexual Medicine guidelines on adult testosterone deficiency, with statements for UK practice. J Sex Med 2017;14:1504-23.

26. Morales A, Bebb RA, Manjoo P, Assimakopoulos P, Axler J, Collier C, et al. Diagnosis and management of testosterone deficiency syndrome in men: clinical practice guideline. CMAJ 2015;187:1369-77.

27. Mulhall JP, Trost LW, Brannigan RE, Kurtz EG, Redmon JB, Chiles KA, et al. Evaluation and management of testosterone deficiency: AUA guideline. J Urol 2018;200:423-32.

28. Dohle GR, Arver S, Bettocchi C, Jones TH, Kliesch S. Male hypogonadism [Internet]. Arnhem: European Association of Urology; c2020 [cited 2020 Aug 4]. Available from: https:// uroweb.org/guideline/male-hypogonadism.

29. Yeap BB, Grossmann M, McLachlan RI, Handelsman DJ, Wittert GA, Conway AJ, et al. Endocrine Society of Australia position statement on male hypogonadism (part 1): assessment and indications for testosterone therapy. Med J Aust 2016;205:173-8.

30. Yeap BB, Grossmann M, McLachlan RI, Handelsman DJ, Wittert GA, Conway AJ, et al. Endocrine Society of Australia position statement on male hypogonadism (part 2): treatment and therapeutic considerations. Med J Aust 2016;205: 228-31.

31. Qaseem A, Horwitch CA, Vijan S, Etxeandia-Ikobaltzeta I, Kansagara D; Clinical Guidelines Committee of the American College of Physicians. Testosterone treatment in adult men with age-related low testosterone: a clinical guideline from the American College of Physicians. Ann Intern Med 
2020; $172: 126-33$

32. Hiort O, Holterhus PM, Horter T, Schulze W, Kremke B, Bals-Pratsch M, et al. Significance of mutations in the androgen receptor gene in males with idiopathic infertility. $\mathrm{J}$ Clin Endocrinol Metab 2000;85:2810-5.

33. Leonard JM, Bremner WJ, Capell PT, Paulsen CA. Male hypogonadism: Klinefelter and Reifenstein syndromes. Birth Defects Orig Artic Ser 1975;11:17-22.

34. Ly LP, Sartorius G, Hull L, Leung A, Swerdloff RS, Wang C, et al. Accuracy of calculated free testosterone formulae in men. Clin Endocrinol (Oxf) 2010;73:382-8.

35. Paller CJ, Shiels MS, Rohrmann S, Basaria S, Rifai N, Nelson W, et al. Relationship of sex steroid hormones with bone mineral density (BMD) in a nationally representative sample of men. Clin Endocrinol (Oxf) 2009;70:26-34.

36. Antonio L, Wu FC, O’Neill TW, Pye SR, Ahern TB, Laurent $\mathrm{MR}$, et al. Low free testosterone is associated with hypogonadal signs and symptoms in men with normal total testosterone. J Clin Endocrinol Metab 2016;101:2647-57.

37. Woods DR, Hill NE, Neely RDG, Talks KL, Heggie A, Quinton R. Hematopoiesis shows closer correlation with calculated free testosterone in men than total testosterone, $\mathrm{J}$ Applied Lab Med 2017;4:441-4.

38. Cohen PG. The hypogonadal-obesity cycle: role of aromatase in modulating the testosterone-estradiol shunt: a major factor in the genesis of morbid obesity. Med Hypotheses 1999;52:49-51.

39. Practice Update. ADA 2020: Testosterone plus lifestyle program reduced risk of type 2 diabetes in overweight and obese men [Internet]. Amsterdam: Elsevier; c2020 [cited 2020 Aug 4]. Available from: https://www.practiceupdate. com/content/ada-2020-testosterone-plus-lifestyle-programreduced-risk-of-type-2-diabetes-in-overweight-and-obesemen/102636.

40. Snyder PJ, Bhasin S, Cunningham GR, Matsumoto AM, Stephens-Shields AJ, Cauley JA, et al. Lessons from the testosterone trials. Endocr Rev 2018;39:369-86.

41. Bhasin S, Cunningham GR, Hayes FJ, Matsumoto AM, Snyder PJ, Swerdloff RS, et al. Testosterone therapy in men with androgen deficiency syndromes: an Endocrine Society clinical practice guideline. J Clin Endocrinol Metab 2010; 95:2536-59.

42. Dalvi M, Walker BR, Strachan MW, Zammitt NN, Gibb FW. The prevalence of structural pituitary abnormalities by MRI scanning in men presenting with isolated hypogonadotrophic hypogonadism. Clin Endocrinol (Oxf) 2016;84:858-
61.

43. El Osta R, Grandpre N, Monnin N, Hubert J, Koscinski I. Hypogonadotropic hypogonadism in men with hereditary hemochromatosis. Basic Clin Androl 2017;27:13.

44. Raju K, Venkataramappa SM. Primary hemochromatosis presenting as type 2 diabetes mellitus: a case report with review of literature. Int J Appl Basic Med Res 2018;8:57-60.

45. Angelopoulos NG, Goula A, Dimitriou E, Tolis G. Reversibility of hypogonadotropic hypogonadism in a patient with the juvenile form of hemochromatosis. Fertil Steril 2005;84: 1744.

46. Gama R, Smith MJ, Wright J, Marks V. Hypopituitarism in primary haemochromatosis; recovery after iron depletion. Postgrad Med J 1995;71:297-8.

47. McDermott JH, Walsh CH. Hypogonadism in hereditary hemochromatosis. J Clin Endocrinol Metab 2005;90:2451-5.

48. National Cancer Institute. Hormone therapy for prostate cancer [Internet]. Bethesda: NIH; 2020 [cited 2020 Aug 4]. Available from: https:/www.cancer.gov/types/prostate/prostate-hormone-therapy-fact-sheet\#: :text=For $\% 20 \mathrm{men} \% 20$ with $\% 20$ intermediate $\% 2$ Drisk, given $\% 20$ for $\% 2018 \%$ E2\%$80 \% 9324 \% 20$ months.

49. Nishikawa E, Jia S, Dharamshi C, Charron V, Lock M. Prostate cancer, gender identity, and testosterone replacement therapy in klinefelter syndrome: a case report and literature review. Cureus 2019;11:e4630.

50. Khoo TK. Adverse events associated with testosterone administration. N Engl J Med 2010;363:1865-6.

51. Warny M, Helby J, Birgens HS, Bojesen SE, Nordestgaard BG. Arterial and venous thrombosis by high platelet count and high hematocrit: 108521 individuals from the Copenhagen General Population Study. J Thromb Haemost 2019;17: 1898-911.

52. Emamian M, Hasanian SM, Tayefi M, Bijari M, Movahedian Far F, Shafiee M, et al. Association of hematocrit with blood pressure and hypertension. J Clin Lab Anal 2017;31:e22124.

53. Muensterman ET, Jaynes HA, Sowinski KM, Overholser BR, Shen C, Kovacs RJ, et al. Effect of transdermal testosterone and oral progesterone on drug-induced qt interval lengthening in older men: a randomized, double-blind, placebo-controlled crossover-design study. Circulation 2019; 140:1127-9.

54. Gravholt $\mathrm{CH}$, Chang S, Wallentin M, Fedder J, Moore P, Skakkebaek A. Klinefelter syndrome: integrating genetics, neuropsychology, and endocrinology. Endocr Rev 2018;39: 389-423. 
55. Quinton R, Tomlinson J. Male hypogonadism and ageing: rejuvenating the guidance. Endocrinologist 2019;131. https:// www.endocrinology.org/endocrinologist/131-spring19/society-news/male-hypogonadism-and-ageing-rejuvenating-theguidance/.

56. Anawalt BD. Diagnosis and management of anabolic androgenic steroid use. J Clin Endocrinol Metab 2019;104:2490500 .

57. Zitzmann M, Faber S, Nieschlag E. Association of specific symptoms and metabolic risks with serum testosterone in older men. J Clin Endocrinol Metab 2006;91:4335-43.

58. Kwong JCC, Krakowsky Y, Grober E. Testosterone deficiency: a review and comparison of current guidelines. J Sex Med 2019;16:812-20.
59. Wu FC, Tajar A, Beynon JM, Pye SR, Silman AJ, Finn JD, et al. Identification of late-onset hypogonadism in middleaged and elderly men. N Engl J Med 2010;363:123-35.

60. Travison TG, Vesper HW, Orwoll E, Wu F, Kaufman JM, Wang $Y$, et al. Harmonized reference ranges for circulating testosterone levels in men of four cohort studies in the United States and Europe. J Clin Endocrinol Metab 2017;102:116173.

61. Boehm U, Bouloux PM, Dattani MT, de Roux N, Dode C, Dunkel L, et al. Expert consensus document: European Consensus Statement on congenital hypogonadotropic hypogonadism: pathogenesis, diagnosis and treatment. Nat Rev Endocrinol 2015;11:547-64. 